\title{
The Laboratory for Laser Energetics' Hydrogen Isotope Separation System
}

\author{
W.T. Shmayda, M.D. Wittman, R.F. Earley, J.L. Reid, and N.P. Redden \\ University of Rochester, Laboratory for Laser Energetics, Rochester, New York, USA
}

\begin{abstract}
The University of Rochester's Laboratory for Laser Energetics has commissioned a hydrogen isotope separation system (ISS). The ISS uses two columns, palladium on kieselguhr and molecular sieve that act in a complimentary manner to separate the hydrogen species by mass. The $4 \mathrm{sL}$ per day throughput system is compact and has no moving parts. The columns and the attendant gas storage and handling subsystems are housed in a $0.8 \mathrm{~m}^{3}$ glovebox. The glovebox uses a helium cover gas that is continuously processed to extract oxygen and water vapor that permeates through the glovebox gloves and any tritium that is released while attaching or detaching vessels to add feedstock to or drawing product from the system. The isotopic separation process is automated and does not require manual intervention. A total of $315 \mathrm{TBq}$ of tritium was extracted from $23.6 \mathrm{sL}$ of hydrogen with tritium purities reaching $99.5 \%$. Deuterium was the sole residual component in the processed gas. Raffinate contained $0.2 \mathrm{TBq}$ of activity was captured for reprocessing. The total emission from the system to the environment was $0.4 \mathrm{GBq}$ over three weeks.
\end{abstract}

Keywords: hydrogen isotope separation, tritium

\section{Background}

The Laboratory for Laser Energetics (LLE) routinely fields $860 \mu \mathrm{m}$ diameter, thin-walled plastic shells filled with deuterium-tritium (DT) gas or DT ice layered on the inner surface of the shells to study inertial confinement physics. The multistep filling operation involves desorbing the DT fuel from uranium storage beds, measuring the tritium inventory, removing decay helium-3 from the DT fuel prior to filling the evacuated shells.

In the case of targets filled with gas, the DT fuel is gradually compressed to pressures in the range of 1 to 3 $\mathrm{MPa}$ in the presence of the shells. DT permeates into the empty shells until the pressure within the shell comes close to equilibrium with the pressure outside the shell. Pressurizing the container holding the shells too quickly can buckle the thin walled plastic spheres. An aluminum coating on the outside of the shells extends the permeation time constant into the tens-of-hours range. At the end of the pressurizing cycle, the DT is returned to the uranium storage beds. The filled targets are transferred to cold storage to further suppress permeation losses from the shells where they are kept until required for experiments.

In the case of cryogenic targets, the DT fuel is assayed, stripped of decay helium-3, and then transferred to a compressor where the gas pressure is first boosted from $0.1 \mathrm{MPa}$ to approximately $12 \mathrm{MPa}$ and subsequently to pressures in the range of 50 to $70 \mathrm{MPa}$ depending on the final ice thickness required. At the end of the pressurization stage, the targets are gradually cooled to $15 \mathrm{~K}$ so that the DT gas that has permeated into the shells can condense on the inner surface of the plastic spheres. At approximately $30 \mathrm{~K}$, gas surrounding the shells is returned to the storage beds. At this temperature gas permeating from the plastic shells is negligible. From this moment onwards these targets are maintained at temperatures between 17 and $40 \mathrm{~K}$ until they are used to study implosion of DT ice driven by the laser system.

These manipulations and time have caused the tritium-to-deuterium ratio to depart from the preferred 50:50 ratio for two reasons over the past few years. First, tritium decays at approximately $0.46 \%$ per month. Second, beta-induced isotopic exchange with hydrogen in the plastic wall and with hydrogen bound in water adsorbed on the inner walls of the process system increases the protium $(\mathrm{H})$ content in the fuel at approximately $0.3 \%$ per year. The first effect leans the fuel to make it deuterium rich. The second effect causes protium to concentrate inside the target and interfere with the implosion kinetics. Both degrade the fusion neutron yield.

\section{Motivation}

The fuel supply has gradually degraded to a tritium/deuterium/protium $(\mathrm{T} / \mathrm{D} / \mathrm{H})$ ratio of 38/59/3. Additionally, during the commissioning of the highpressure systems, LLE used $48 \mathrm{TBq}(1300 \mathrm{Ci})$ of tritium to make up gas mixtures ranging from $0.1 \% \mathrm{~T} / \mathrm{D}$ to $10 \%$ $\mathrm{T} / \mathrm{D}$. This gas is not useful as a fuel and has been stored on a separate uranium storage bed that is not connected to any of the existing gas handling systems. Finally, LLE has installed emission reduction equipment based on getter technology that will be discussed shortly. Elemental tritium can be extracted from helium purge stream with a very high efficiency ${ }^{1}$ with getters. While these getters can be regenerated to recover elemental tritium with negligible release to the environment, the gas will be strongly contaminated with protium,

author's email: wshm@lle.rochester.edu 
rendering it useless for inertial confinement physics studies.

LLE operates under a $1.5 \mathrm{~g}$ tritium inventory limit. The majority of this gas is required for the operations discussed in the previous section. LLE does not have the inventory headroom to replace downgraded tritium with fresh tritium without first disposing of the downgraded tritium off-site. For example, an accidental release of $30 \%$ of LLE's tritium inventory and its subsequent recovery as downgraded tritium would preclude LLE from undertaking any further inertial fusion confinement studies until the downgraded tritium was either disposed as waste or treated to recover the tritium. Disposing of elemental tritium gas is prohibitively expensive and not a viable approach; isotopic separation is more attractive. Furthermore, outfitting LLE with an isotopic separation system expands the Laboratory's capabilities to explore fusion reaction physics over a very broad range of $T / D$ ratios in a controlled manner.

A review of the hydrogen isotope separation technologies suggested that a scaled down version of the 'thermal cycling absorption process' known as TCAP ${ }^{2}$ developed at Savannah River National Laboratory would be ideally suited to meet LLE's needs. The system in its most recent re-incarnation offers several unique advantages. The system has no moving parts with the exception of one automated valve. Gases are shuffled within the system by heating or cooling storage beds or columns. This increases the system's robustness against accidental release of tritium.

The system is compact. Atomic sorting of the hydrogen species by mass occurs in the presence of palladium on kieselguhr $(\mathrm{Pd} / \mathrm{k})$. Molecular hydrogen isotopologue sorting by mass occurs on cold molecular sieve (MS). Both processes are complimentary and reinforce the movement of the heaviest species to the front end of the $\mathrm{Pd} / \mathrm{k}$ column while the lightest species prefer to accumulate at the exhaust end of the MS column.

Emission from the system is controlled by adjusting the operating conditions of the ISS and the number of times the gas is shuffled between the two columns. Additionally ISS raffinate effluents with measureable activity can be directed to and concentrated in the Glovebox Cleanup System as hydrides and subsequently returned to the ISS for tritium recovery.

\section{Description of LLE's isotope separation system}

\subsection{Overview}

An overview of the LLE isotope separation system is provided in figure 1. The complete system is made up of five subsystems: Gas Handling, Core System, LN2 management, Vacuum, and Glovebox Cleanup. The Gas Handling System feeds isotopically diluted tritium to the Core System and provides a temporary storage capability for purified tritium. The Core System decomposes the mixed hydrogen isotopes and separates them to isolate pure tritium. The LN2 system provides a high cooling capability for the $\mathrm{Pd} / \mathrm{k}$ and MS columns. The Vacuum system supports all evacuation steps required in handling tritium within the Gas Handling and Core Systems. The Glovebox Cleanup System provides secondary containment for any tritium releases from the Gas Handling and Core Systems. Only the Gas Handling System and the Core are housed inside the glove box.

\subsection{Gas Handling System}

The Gas Handling System comprises a uranium storage bed, a palladium storage bed and a valve tree for diagnostics and for transferring gas into and out of the Core. Swagelok BNBW Series Bellows valves fitted with copper stem tips were used throughout. The valve tree is an integrated, welded assembly with a global leak rate below $1 * 10^{-9} \mathrm{~atm}-\mathrm{cc} / \mathrm{s}$. The valves are rated to operate from vacuum to $3.45 \mathrm{MPa}$ (500 psig) spanning a temperature range from $283 \mathrm{~K}$ to $477 \mathrm{~K}$. Swagelok destructively tested a set of seven $\mathrm{BN}$ valves at their factory in support of this project. The burst or fail pressure of these valves is $90 \mathrm{MPa}$ (13,070 psig)

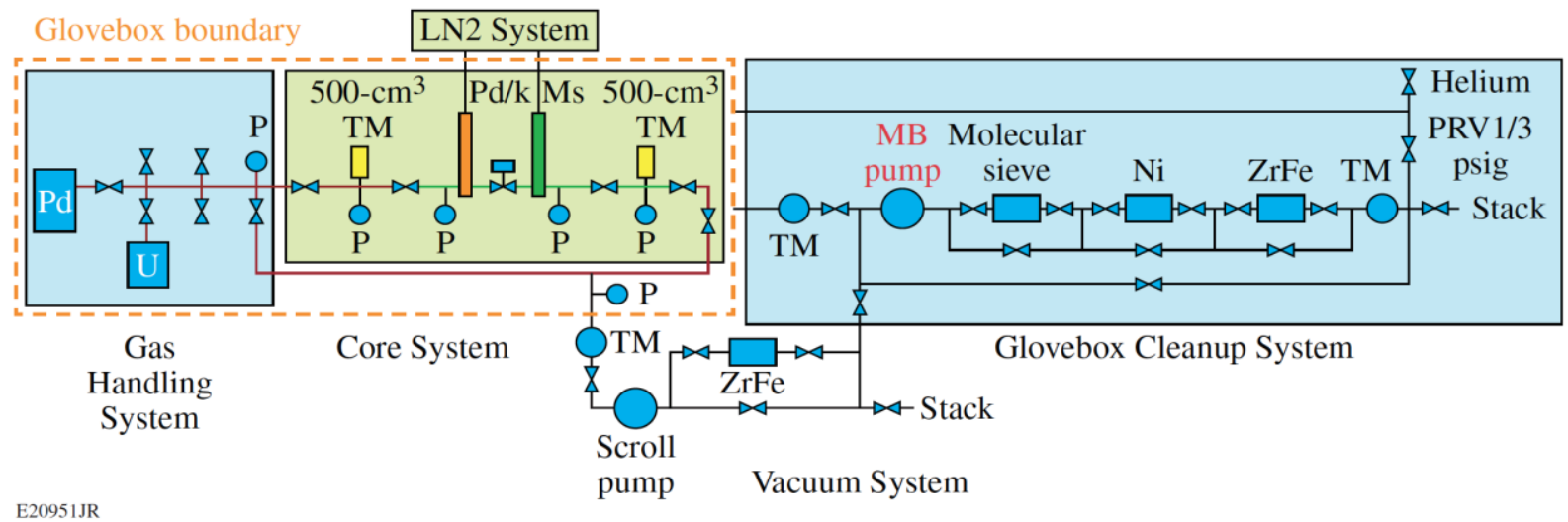

Notation: $\mathrm{Pd}=$ palladium bed, $\mathrm{U}=$ uranium bed, $\mathrm{TM}=$ tritium monitor, $\mathrm{Pd} / \mathrm{k}=$ palladium on keiselghur, $\mathrm{MS}=$ molecular sieve, $\mathrm{MB}=$ metal bellows, $\mathrm{Ni}=$ nickel bed, $\mathrm{ZrFe}=$ zirconium-iron ally bed, $\mathrm{PRV}=$ pressure relief valve.

Fig. 1. Overview of the LLE isotope separation system. 
when closed and $32.5 \mathrm{MPa}$ (4700 psig) when open. The stem bellows twist and relieve the pressure when the valve is open.

MKS 870 series Baratron pressure transducers were deployed in both the Gas Handling and Core Systems. The burst pressure of these transducers was measured to be $132 \mathrm{MPa}(19,140 \mathrm{psig})$.

The uranium storage bed contains $34.1 \mathrm{~g}$ of depleted uranium and features a flow-through configuration to permit circulation over the uranium powder and is doublycontained to reduce heat and tritium losses to the glovebox when the bed is heated. The bed was designed and built to ASME VIII Div 1 and is registered as a pressure vessel. The maximum allowable working pressure is $1.48 \mathrm{MPa}(200 \mathrm{psig})$ at $600 \mathrm{~K}$ for the primary vessel and $0.45 \mathrm{MPa}$ (50 psig) at $393 \mathrm{~K}$ for the secondary vessel. The hydrogen storage capacity is $4.5 \mathrm{sL}$.

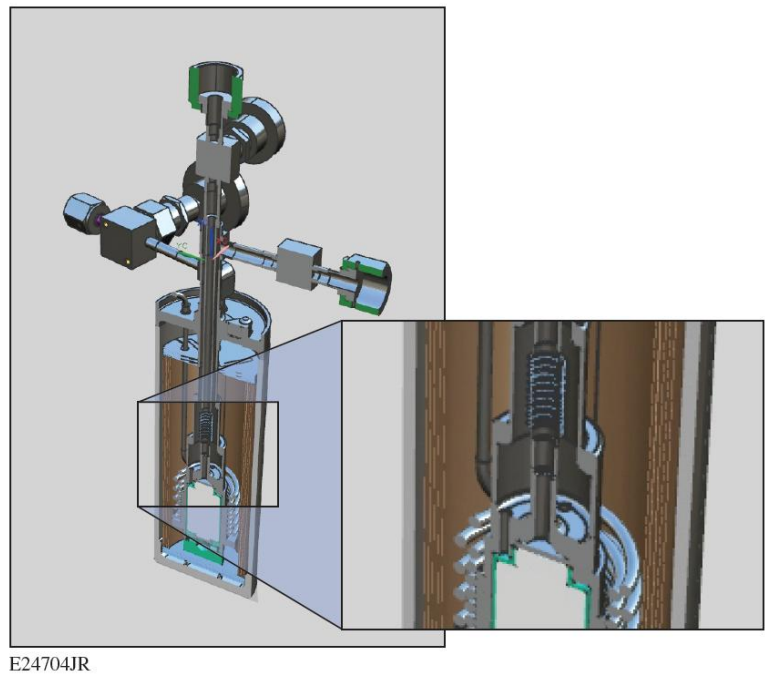

Fig. 2. Cross-section of the flow through uranium storage bed used in the Gas Handling System.

A cartoon of the cross-section of the uranium storage bed is provided in figure 2. In purge mode, gas enters via the upright valve to flow into the uranium powder that is trapped between two $5 \mu \mathrm{m}, 1.6 \mathrm{~mm}$ thick stainless steel frits, exits via the lower filter, passes through channels cut into the inside of the primary vessel wall and flows along an annular region of the central tube before exiting the bed via the valve positioned at $90^{\circ}$ relative to the central tube. The central tube incorporates a formed bellows to relieve stress between the primary and secondary containers when the bed is heated to $600 \mathrm{~K}$. The annular region between the primary and secondary containers is kept under vacuum for most operations using the bed.

The palladium storage bed design parallels that of the uranium bed. It contains $63.1 \mathrm{~g}$ of palladium and also features a flow through design and utilizes double containment. The maximum allowable working pressure is $1.48 \mathrm{MPa}$ (200 psig) at $483 \mathrm{~K}$. The storage capacity of the Pd bed is $5.7 \mathrm{sL}$. The Pd bed is designed to operate between $120 \mathrm{~K}$ and $425 \mathrm{~K}$. To achieve subzero temperatures, an acoustic Stirling (pulse tube) cryocooler $^{3}$ is thermally coupled to the Pd bed via a cold finger joint that separates at elevated temperatures. The pressurewave generator and the compliance tank with an inertance tube coil reside outside the glovebox while the primary containment vessel of the Pd bed is located inside the glovebox. The configuration is shown in figure 3 .

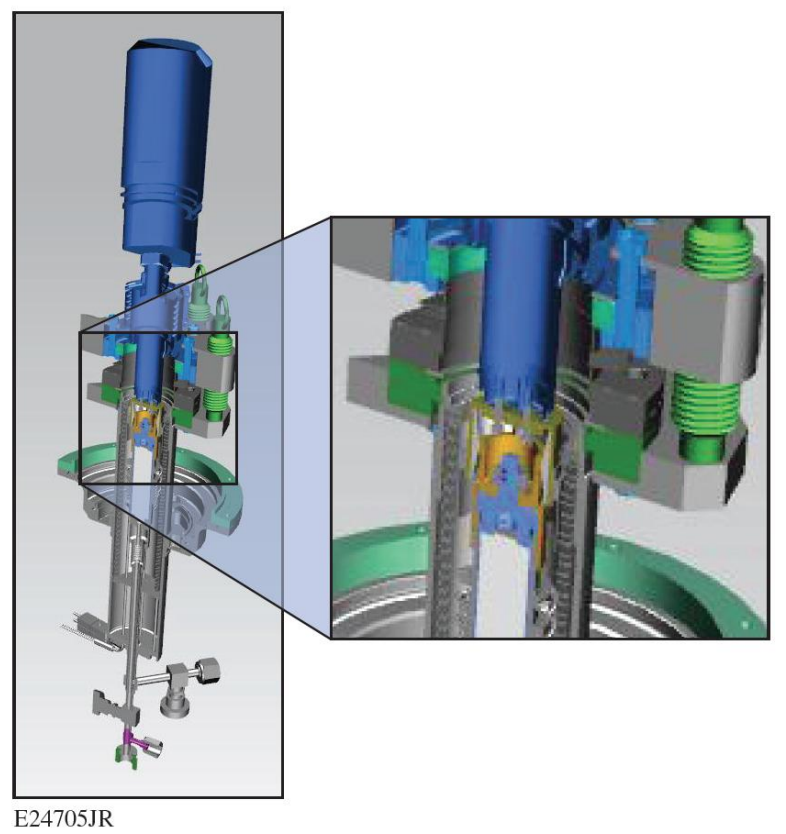

Fig. 3. Cross-section of the flow through palladium storage bed used in the Gas Handling System. The flange welded to the Pd bed secondary containment seals against the glovebox ceiling to isolate the cryocooler from the glovebox atmosphere.

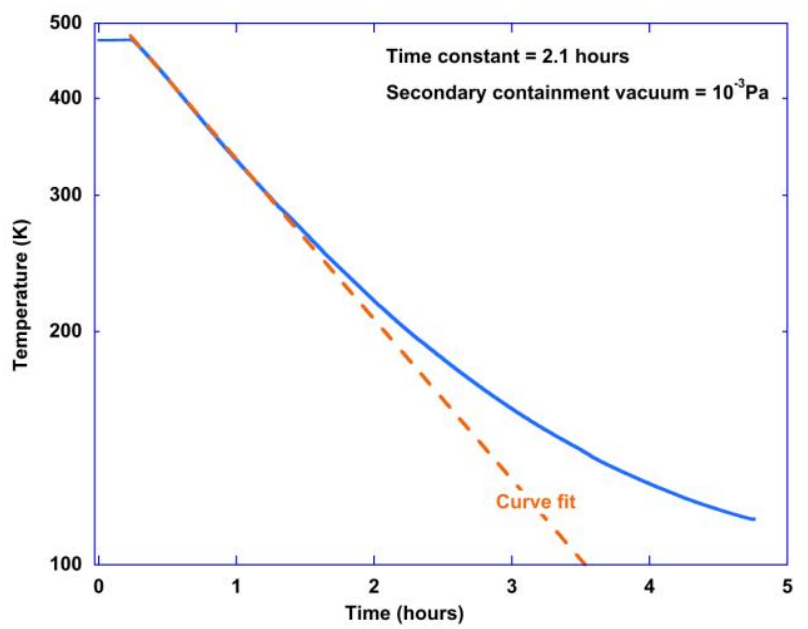

Fig. 4. Pd bed primary vessel cool down rate dependence on the secondary containment vacuum.

This cooling arrangement provides fine temperature control and simplifies the liquid nitrogen cooling circuit inside the glovebox. Heat loss to the secondary containment determines the Pd bed cool down rate as well as the lowest attainable temperature. As illustrated in figure 4, when the secondary containment vacuum is $10^{-3}$ $\mathrm{Pa}$, the $\mathrm{Pd}$ bed temperature drops exponentially with a 2.1 
hour time constant for the first hour and then at a slower rate to reach an operating temperature of $157 \mathrm{~K}$ in three hours.

\subsection{Core System}

The Core System uses two columns, palladium on kieselguhr $(\mathrm{Pd} / \mathrm{k})$ and molecular sieve $(\mathrm{MS})$, separated by a low-volume, diaphragm valve. An exploded cartoon of a column assembly is illustrated in figure 5. The column assembly is made by coiling the column comprising 6.4 $\mathrm{mm}(1 / 4$ ") diameter stainless-steel tubing filled with $\mathrm{Pd} / \mathrm{k}$ and sandwiching the column between a coiled heater on one side and a $6.4 \mathrm{~mm}$ diameter LN2 cooling coil on the opposite side. The three coils are brazed together to improve heat transport while the column is thermally cycled using liquid nitrogen cooling and electrical heating. High-density insulation is inserted between the column assembly and the column end covers to reduce heat losses to the glovebox. The burst pressure of the column is $158 \mathrm{MPa}$ (22940 psig).

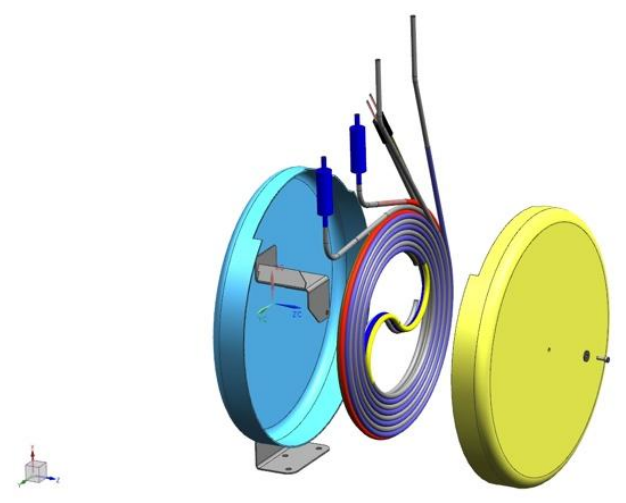

Fig. 5. Exploded view of one column used the Core System. Both columns are similar in design differing only in length.

A modified, all-metal, low-volume Swagelok 6LVVDP series valve close couples the $\mathrm{Pd} / \mathrm{k}$ and $\mathrm{MS}$ column assemblies. The burst pressure of this valve is $84.2 \mathrm{MPa}$ $(12,200 \mathrm{psig})$. The valve is oriented in the circuit to relieve gas pressure from the MS column into the $\mathrm{Pd} / \mathrm{k}$ should the system inadvertently lose LN2 cooling capability.

The pressure transducers are high-pressure MKS Series 870 Baratron sensors described in Section 3.2.

The expansion vessels double as tritium monitors. The design is based on the one-liter process monitor ${ }^{4}$. The cylindrical wall length was shortened to reduce the internal volume of the chamber to $500 \mathrm{cc}$. The end caps have been thickened to help support the floating BNC used to measure the ionization current. The monitor is registered under ASME VII Div 1. With a burst pressure of $33 \mathrm{MPa}$ (4800 psig), the tritium monitor represents the weakest link in the Core System. The two monitors operate from vacuum to $3.6 \mathrm{MPa}$ (500 psig) over the temperature range $260 \mathrm{~K}$ to $360 \mathrm{~K}$. The internal surfaces were gold coated to reduce the memory effects of the monitor [4]. (a)

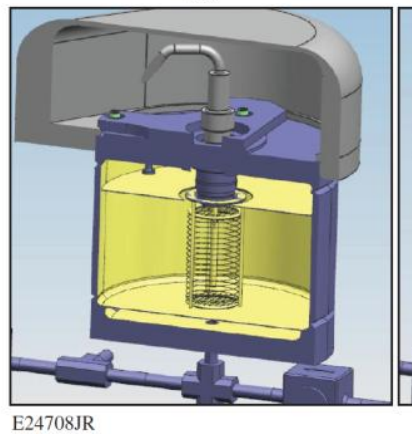

Fig. 6. The cross-section of the expansion vessel/tritium monitor (a) on the product side is shown on the left. The cross-section of the expansion vessel/tritium monitor (b) on the raffinate side is shown on the right. Both devices are used in the Core System.

Cross-sections of the two expansion vessels are illustrated in figure 6 . The collector within the raffinate monitor is a solid cylindrical configuration. The outer shell of the monitor is at zero potential. The ionization current induced by tritium decay within the detection region increases linearly with tritium activity of the range $74 \mathrm{kBq} / \mathrm{m}^{3}$ to $37 \mathrm{GBq} / \mathrm{m}^{3}$ for flow rates below $40 \mathrm{sLPM}$ and is practically independent of operating pressures above $9 \mathrm{kPa}$ as discussed in reference 4 .

The collector in the product monitor is a wire cage housed at the center of the $500 \mathrm{~cm}^{3}$ expansion vessel. The anode is approximately $3 \mathrm{~mm}$ in diameter. The detection volume is $20 \mathrm{~cm}^{3}$. The minimum detectable tritium activity in helium is $4 \mathrm{MBq} / \mathrm{m}^{3}$ when this detector is coupling to a one femto-ampere transimpedance amplifier.

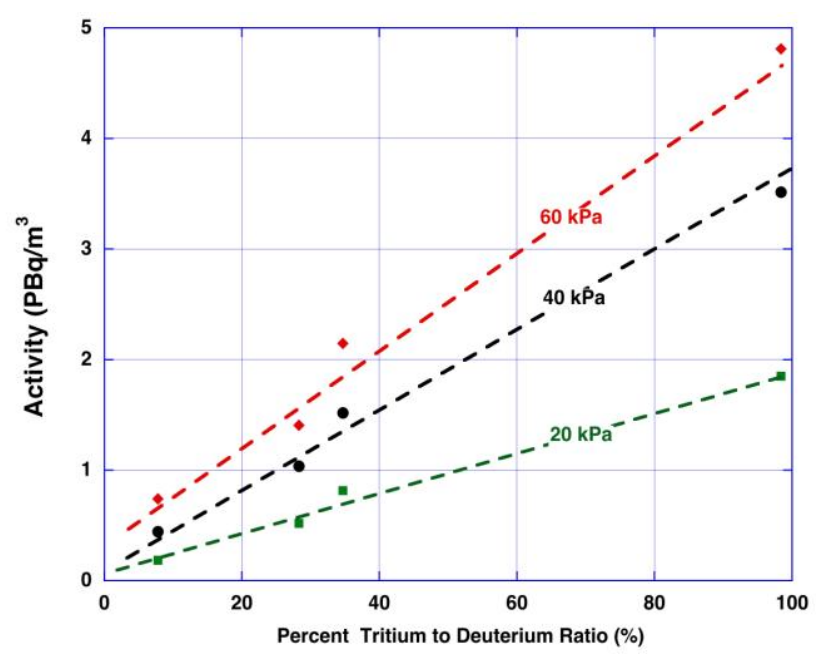

Fig 7. Response of the $20-\mathrm{cm}^{3}$ wire-cage ionization chamber to tritium activity at fixed total pressures.

The saturation current dependence on tritium concentration for fixed total pressures in the $20 \mathrm{cc}$ wire cage detector increases linearly with tritium content over the pressure range 20 to $60 \mathrm{kPa}$ (150 to 450 Torr) as shown in figure 7 . However, figure 8 indicates that the increase in the saturation current with increasing pressure for different tritium activities in deuterium is less than linear for the $20 \mathrm{~cm}^{3}$ detector. Evidently electron-ion recombination in the gas becomes progressively more significant as the total pressure in the chamber increases. 


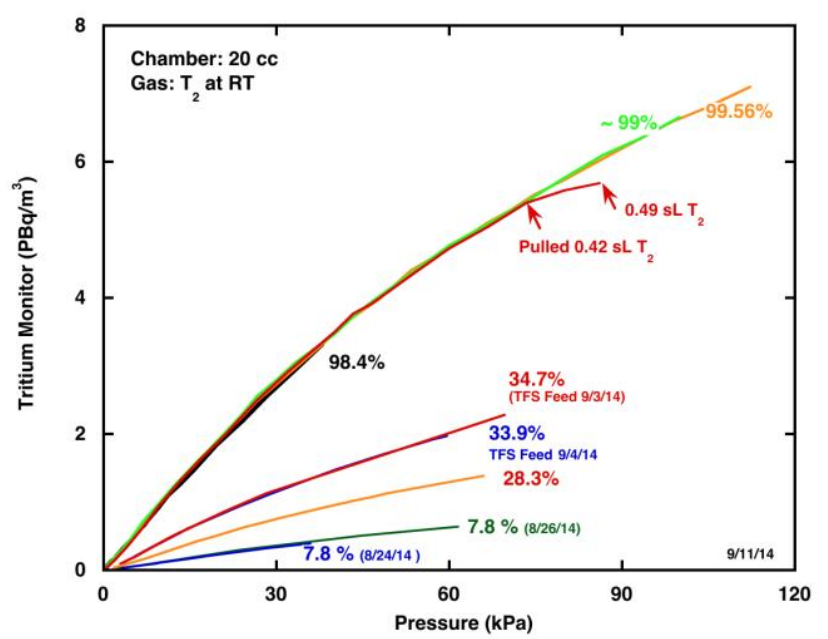

Fig. 8. Response of the $20-\mathrm{cm}^{3}$ wire-cage ionization chamber to increasing total pressure for different concentrations of tritium in deuterium.

\subsection{LN2 System}

The thermal ramp of the columns in the Core System needs to be in the vicinity of 0.5 to $1 \mathrm{~K} / \mathrm{s}$ for good separation. A liquid nitrogen cooling circuit was installed to achieve this precipitous temperature swing. A 350-liter dewar is housed in a separate room that is ventilated directly to the room exhaust ventilation duct. Liquid nitrogen is transferred to the columns via vacuumjacketed lines. Gaseous nitrogen is directed to the room exhaust via a second set of vacuum-jacketed lines. Several automated responses have been designed into the LN2 System to address off-normal events. These include oxygen-deficiency monitors; system isolation if the cooling rate deviates from the expected trajectory; an independent, centralized monitoring station; and pagers to notify key personnel if parameters depart from design values.

\subsection{Vacuum System}

The vacuum system is manually isolated from the Gas Handling System by two Swagelok 1/2”, 8 B-series valves fitted with copper stem tip valves to reduce the potential for inadvertent evacuation of high-activity gas from the process loop. A one-liter expansion vessel that also serves as a tritium detector separates the two manual valves. Vacuum is generated by a conventional turbomolecular/scroll pump combination.

Evacuation of the Gas Handling System is done under stringent safety protocols to prevent the release of tritium into the environment. Safety protocols require operators to pre-evacuate the one-liter detector, isolate the detector from the vacuum system, expand the contents of the Gas Handling System into the tritium monitor, assay the activity of the gas and then direct the gas in one of three directions. The effluent can be:

- discharged directly to the room exhaust ventilation duct,

- passed over an ST198 alloy to reduce the amount of effluent discharged to the room exhaust, or
- injected into the Glovebox Cleanup System for additional treatment after a preliminary pass over the ST198 alloy.

\subsection{Glovebox Cleanup System}

The Glovebox Cleanup System is a stand-alone recovery system used to extract tritium from gloveboxes that use helium gas as the working atmosphere. ${ }^{5,6,7}$ The system is built around ST198 alloy from SAES Getters located in Milan, Italy. Elemental hydrogen forms a hydride with this alloy. The process is reversible, so that periodically the alloy can be regenerated to recover tritium gas and to return the 'emptied' bed back into service.

Typically the life expectancy of such a bed is several years before the capacity of the alloy is reduced to unacceptable levels by oxidation. Both water vapor and oxygen permeate into the glovebox from the atmosphere via the rubber gloves. Both gases irreversibly consume the ST198 alloy.

The elements of the Glovebox Cleanup System are illustrated in figure 1. Gas is extracted from the glovebox with a Senior Flexonics' MB158 diaphragm pump through a tritium monitor and discharged into a train of three getters: a molecular sieve drier to remove water vapor, a nickel bed to remove oxygen, and the ST198 bed. Treated gas is returned to the glovebox via a second tritium monitor. The box pressure is adjusted to remain about $2 \mathrm{~Pa}$ below the ambient pressure. Treated helium can be discharged to the environment just ahead of a $2 \mathrm{kPa}$ pressure relief valve (PRV) to reduce the box pressure or fresh helium can be added to the box downstream of the PRV to increase the box pressure.

\section{Isotope Separation System Operations}

\subsection{Commissioning}

The performance of the Core System was evaluated using hydrogen/deuterium gas mixtures. The two hydrogen isotopes were introduced to the product side (front end) of the $\mathrm{Pd} / \mathrm{k}$ coil in varying ratios from gas bottles. Deuterium was condensed on the $\mathrm{Pd} / \mathrm{k}$ column cooled with liquid nitrogen first. Then hydrogen was added to push the deuterium deeper into the column. Subsequently the gas mixture was shuffled between the $\mathrm{Pd} / \mathrm{k}$ and the Molecular Sieve (MS) columns by heating the sending column while cooling the receiving column. The gas was transferred back and forth via the valve interconnecting the two columns illustrated in figure 1. A typical cycle to shuffle the hydrogen from the sending column to the receiving column and back again required about 1 hour. Typically 20 to 24 cycles were used to separate the two isotopologues of hydrogen. Product was eluted in measured aliquots from the front end of the $\mathrm{Pd} / \mathrm{k}$ column until mixed isotopologues of hydrogen appeared. Subsequently the gas was transferred to the MS column and drawn from the back end of the MS column as raffinate. The composition of both the product and raffinate gas as a function of the quantity of gas extracted 
from either end was measured using a thermal conductivity detector.

An example of product $\left(D_{2}\right)$ drawn from the $\mathrm{Pd} / \mathrm{k}$ column is illustrated in figure 9 . This figure indicates that the first liter of gas drawn from the $\mathrm{Pd} / \mathrm{k}$ column is pure $\mathrm{D}_{2}$. The detection limit of the thermal conductivity detector is estimated to be $0.005 \%$. The following $80 \mathrm{scc}$ of gas comprises $62.4 \% \mathrm{H}_{2}, 30.3 \% \mathrm{HD}$ with the balance being $\mathrm{D}_{2}$. The second $80 \mathrm{scc}$ aliquot that is drawn consists of $77.2 \% \mathrm{H}_{2}$ and $21.1 \% \mathrm{HD}$. The composition of the final $440 \mathrm{scc}$ drawn is $80.8 \% \mathrm{H}_{2}$ and $18.0 \% \mathrm{HD}$. In this example, the $\mathrm{Pd} / \mathrm{k}$ column was charged to $90 \%$ of its capacity with a mixture comprising $27 \% \mathrm{D}_{2}$ and $73 \% \mathrm{H}_{2}$ and cycled 24 times before product was drawn from the front end of the $\mathrm{Pd} / \mathrm{k}$ column. Within the detection limit of the thermal conductivity detector, $67 \%$ of the deuterium introduced into the column as a mixture was recoverable as isotopically pure $\mathrm{D}_{2}$. This extraction efficiency was typical in all cases investigated when the system operating conditions were set to yielded pure product. It is noteworthy that palladium absorbs elemental hydrogen as atoms and that the atoms need to recombine with their nearest neighbors to desorb from the palladium. Consequently recovering a $27 \% \mathrm{D}_{2}$ and $73 \%$ $\mathrm{H}_{2}$ load from palladium without cycling would be isotopically scrambled to form a mixture comprising $<1 \%$ $\mathrm{D}_{2}, \sim 27 \% \mathrm{HD}$ and $\sim 73 \% \mathrm{H}_{2}$.

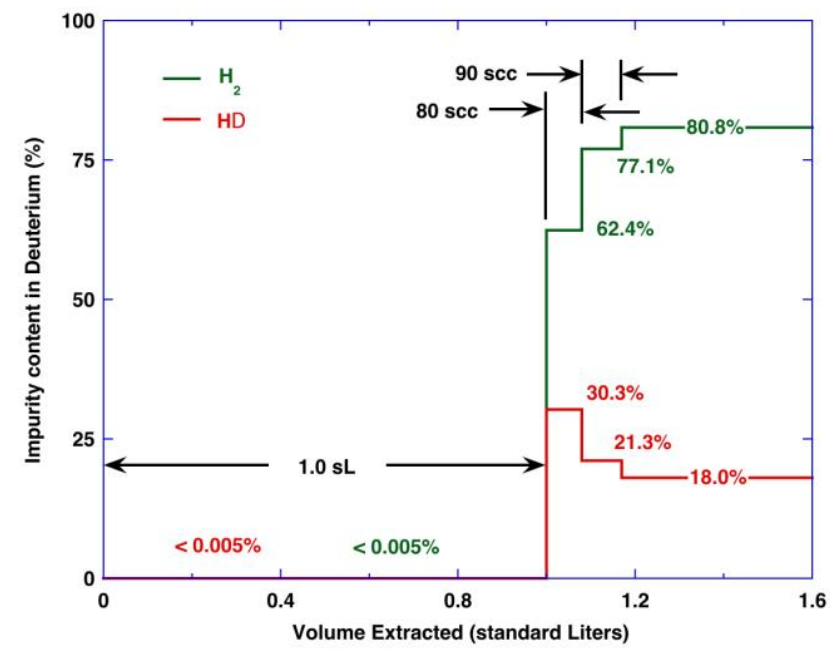

Fig. 9. Composition of product drawn from the $\mathrm{Pd} / \mathrm{k}$ column following 24 cycles. The initial charge was a mixture comprising $27 \% \mathrm{D}_{2}$ and $73 \% \mathrm{H}_{2}$ mixture.

Figure 9 also indicates that the transition from pure product to mixed isotopes is extremely sharp. This behavior was typical of all runs that generated pure product.

Increasing the gas inventory to $98 \%$ of the capacity of the $\mathrm{Pd} / \mathrm{k}$ column degraded the performance of the system. The product was contaminated with up to $1.1 \%$ of protium (H). Similarly decreasing the gas inventory to $82 \%$ of the capacity of the $\mathrm{Pd} / \mathrm{k}$ column also degraded system performance. Figure 10 illustrates the gas composition of the product when the system is operated at $82 \%$ of the capacity of the $\mathrm{Pd} / \mathrm{k}$ coil.

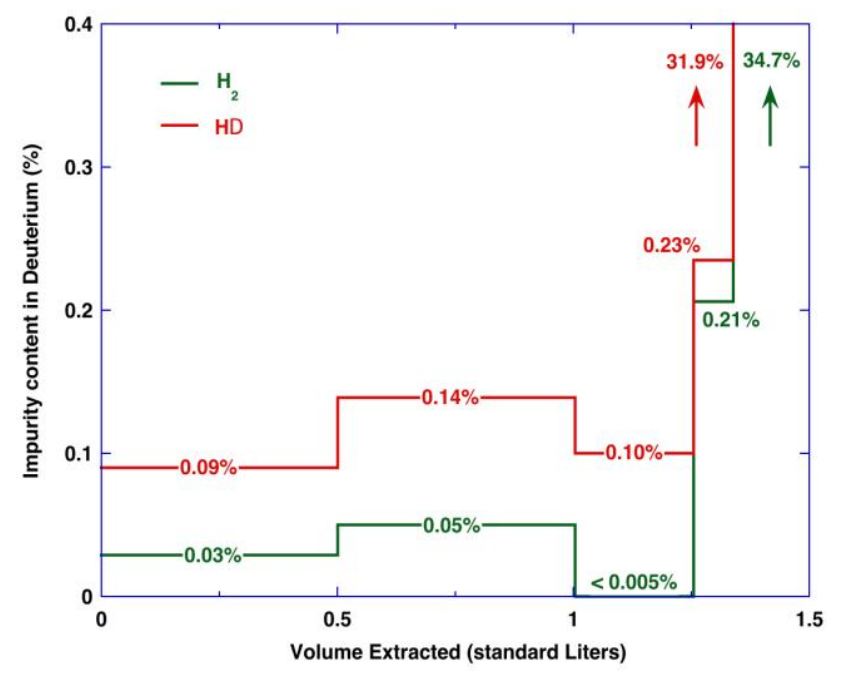

Fig. 10. Composition of product drawn from the $\mathrm{Pd} / \mathrm{k}$ column after 20 cycles. The initial charge was a mixture comprising $40 \% \mathrm{D}_{2}$ and $60 \% \mathrm{H}_{2}$.

The product was contaminated with $0.03 \%$ hydrogen in the first half liter and $0.05 \%$ hydrogen in the second half liter even though the $\mathrm{D}_{2} / \mathrm{H}_{2}$ ratio was higher than in the mixture shown in figure 9 . It appears that column performance is optimal when operating around $90 \%$ of the $\mathrm{Pd} / \mathrm{k}$ column capacity.

Following the extractions discussed in figure 10, the remaining gas was transferred to the MS coil and discharged as raffinate. The composition of the raffinate is provided in figure 11 .

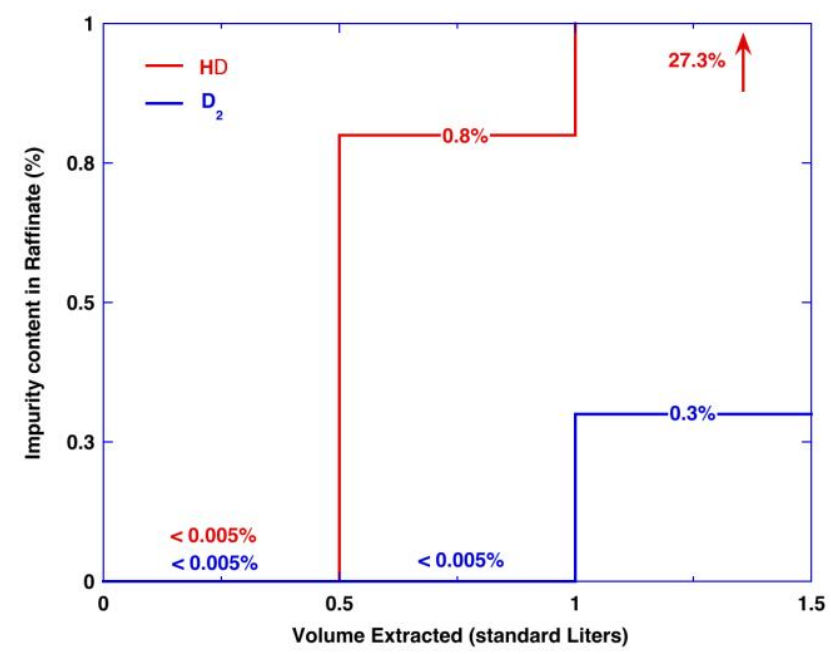

Fig. 11. Composition of raffinate drawn from the exhaust end of the MS column after 20 cycles and extracting $1.5 \mathrm{sL}$ of product from the $\mathrm{Pd} / \mathrm{k}$ coil. The initial charge was a mixture comprising $40 \% \mathrm{D}_{2}$ and $60 \% \mathrm{H}_{2}$.

Even though the performance of the ISS was not optimal while operating at $82 \%$ of the capacity of the $\mathrm{Pd} / \mathrm{k}$ column, the first half-liter of raffinate extracted from the MS coil did not contain any deuterium. This example implies that raffinate containing little if any tritium can be drawn when separating tritium from hydrogen, deuterium and tritium mixtures. 


\subsection{Processing Active Gas}

The purification of LLE's fuel supply proceeded by gradually increasing the quantity of tritium present in the ISS. Initially, a lower activity hydrogen, formed by blending $7.75 \%$ tritium-in-hydrogen with pure deuterium, was used to verify the leak integrity of the various subsystems, confirm that all detectors were operational and vet the operational procedures. Subsequently, aliquots of hydrogen containing $7.75 \%$ tritium were added to the ISS to replace the inactive raffinate that was removed from the system. Finally, eight liters of hydrogen containing 34\% tritium were added to the ISS. Over the course of three weeks, $23.6 \mathrm{sL}$ of tritiated hydrogen were processed. Raffinate contained $0.2 \mathrm{TBq}$ of activity was captured on a zirconium-iron scavenger bed and recovered for tritium extraction. Raffinate containing very small amounts of tritium was discharged directly to the environment. The total emission from the system to the environment was $0.4 \mathrm{GBq}$ over the three-week processing period.

Figure 12 illustrates the isotopic composition of one of the feed streams processed by the ISS. The protium $(\mathrm{H})$ component makes up $5.45 \%$ of the gas mixture partly as elemental hydrogen and partly as protium-containing isotopologues of deuterium and tritium.

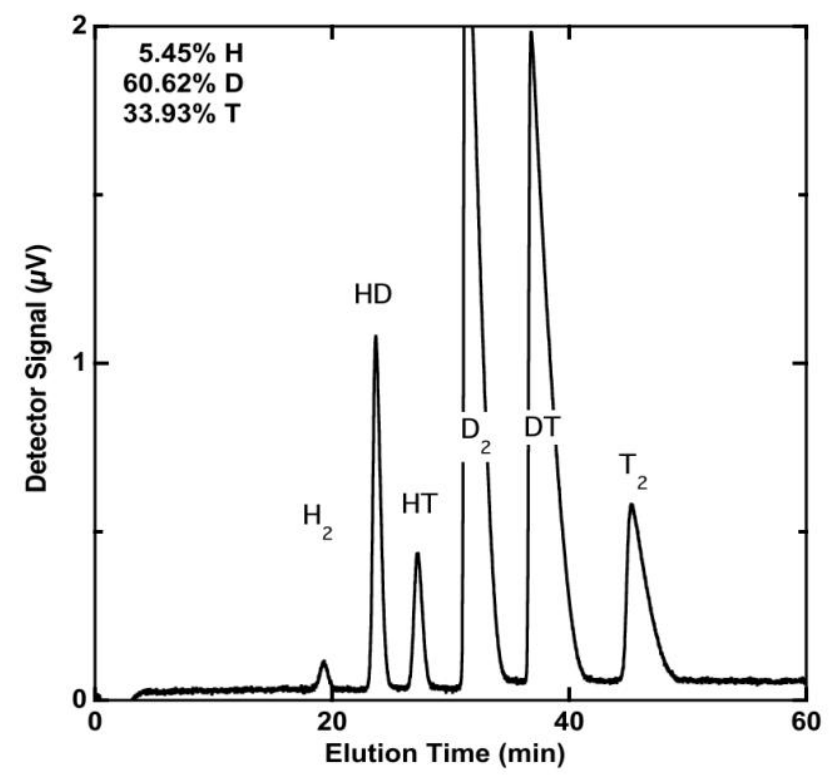

Fig. 12. Isotopic composition of one of the feedstock of hydrogen processed in the ISS.

Figure 13 illustrates the isotopic composition of purified tritium. Protium is not observed within the detection limit of the thermal conductivity detector even though the sensitivity of the detector has been increased seven fold. The presence of deuterium in the mixture was not a concern because this gas was subsequently blended with purified deuterium to make a $65 / 45 \mathrm{~T} / \mathrm{D}$ gas mixture that was used to fill targets and study inertial confinement D-T fusion physics.

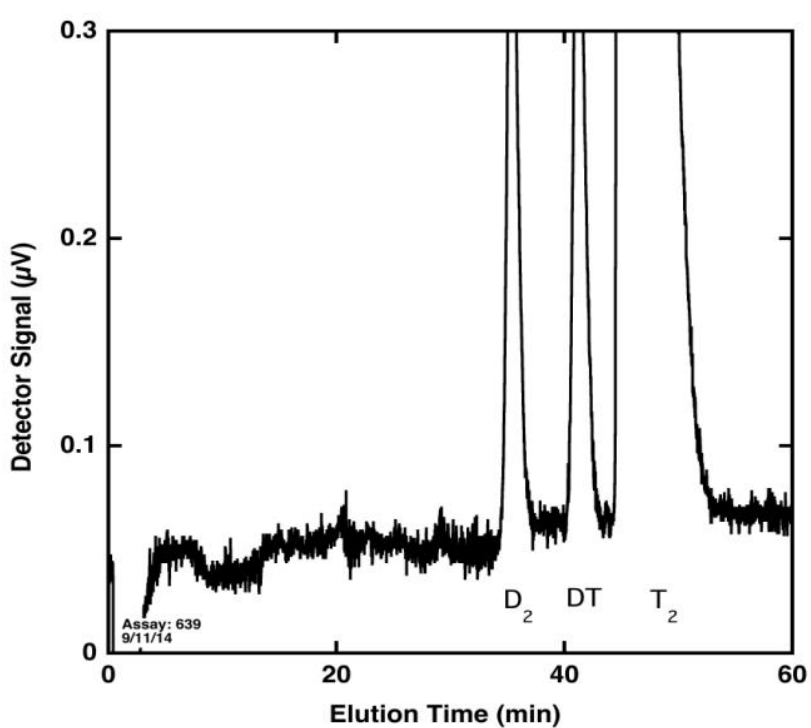

Fig. 13. Isotopic composition of purified tritium drawn from the front end of the $\mathrm{Pd} / \mathrm{k}$ column.

\section{Acknowledgments}

The design and testing of the Core System was carried out in collaboration with Drs. S. Xiao and L.K. Heung, Mr. T. Sessions and Mrs. S. Redd of Savannah River Site (SRNL). The software to control of the Core System was provided by SRNL. The development of the system proceeded at a frenetic pace to meet LLE's deadline. The professionalism of SRNL and LLE was exemplary.

This material is based upon research work supported by the U.S. Department of Energy National Nuclear Security Administration under award DE-NA0001944, the University of Rochester, and the New York State Energy Research and Development Authority.

\section{References}

\footnotetext{
1 W. T. Shmayda, N. P. Kherani, and A. G. Heics, Tritium Removal From Noble Gas Streams, J. Vac. Sci. Technol. A 6, 1259 (1988).

${ }^{2}$ S. Xiao, L. K. Heung, and H. T. Sessions, Recent Advances on Hydrogen Isotope Separation using Thermal Cycling Absorption Process, Fusion Science and Technology, Vol. 67, 2015, pp 643-6.

3 J. F. Burger, H. J. M. ter Brake, H. Rogalla, and M. Linder, Vibration-free 5K sorption cooler for ESA's Darwin mission, Cryogenics, 42 (2002) 97-108.

${ }^{4}$ N. P. Kherani and W. T. Shmayda, In-Line Process Tritium Monitor, Fusion Technol., 21, 340 (1992).

${ }^{5}$ N. P. Kherani, W. T. Shmayda, and R. A. Jalbert, Tritium Removal from Inert Gases Using $\mathrm{Zr}_{2} \mathrm{Fe}$, IEEE 87 CH2507, 1239 (1987).

6 W. T. Shmayda, N. P. Kherani, B. Wallace, and F. Mazza, Inert Gas Secondary Enclosure Clean-Up System, Fusion Technol. 2, 616 (1992).

7 A. G. Heics and W. T. Shmayda, Development of a Secondary Enclosure Clean-Up System for Tritium Systems, IEEE 93CH3348-0, 65 (1993).
} 